\title{
Feasibility Study of Transcatheter Arterial Chemoembolization with Epirubicin Drug-eluting Beads for Hepatocellular Carcinoma in Japanese Patients
}

1) Department of Diagnostic Radiology, National Cancer Center, Japan 2) Department of Diagnostic Radiology, Aichi Cancer Center, Japan

Hiroaki Ishii $^{1)}$, Yasuaki Arai $^{1)}$, Miyuki Sone ${ }^{1)}$, Shunsuke Sugawara ${ }^{1)}$, Shinichi Morita ${ }^{1)}$, Yoshitaka Inaba ${ }^{2)}$

\section{Abstract}

Purpose: To evaluate the feasibility of drug-eluting bead (DEB)-transarterial chemoembolization (TACE) with $75 \mathrm{mg}$ epirubicin for hepatocellular carcinoma (HCC) in Japanese patients with unresectable HCC prior to conducting a planned randomized controlled trial.

Materials and Methods: This study was conducted as a prospective multi-center feasibility study. Eligible patients had unresectable Barcelona Clinic Liver Cancer stage A or B HCC that was unsuitable for curative treatments, and all patients received TACE with $75 \mathrm{mg}$ epirubicin-loaded DEB. Tumor response, as the primary endpoint, was assessed after 4 weeks by computed tomography or magnetic resonance imaging, based on the modified Response Evaluation Criteria in Solid Tumors. Adverse events after treatment were evaluated as the secondary endpoint, based on the Common Terminology Criteria for Adverse Events version 4.0.

Results: Between May and August 2014, 8 patients from two institutions were enrolled in this clinical study. There were no instances of complete response observed, partial response was obtained in 4 patients, and the overall response rate was 50\%. No patients experienced grade 4 or higher adverse events. Grade 3 thrombocytopenia occurred in 1 patient. One patient experienced a grade 3 increase in aspartate aminotransferase, alanine aminotransferase, and bilirubin levels. All adverse events were well managed with conservative medical care. There were no procedure-related deaths.

Conclusions: DEB-TACE with $75 \mathrm{mg}$ epirubicin was found to be feasible in Japanese patients, and it was deemed appropriate to proceed to a randomized controlled trial comparing DEB-TACE and conventional TACE.

Key words: Transcatheter Arterial Chemoembolization, Hepatocellular Carcinoma, Drug-eluting Beads

(Interventional Radiology 2017; 2: 79-84)

\section{Introduction}

For unresectable hepatocellular carcinoma (HCC), transarterial chemoembolization (TACE) has become an established treatment with level I evidence derived from randomized controlled trials (RCTs) and meta-analyses [1-3]. Fundamental elements of TACE include administration of a chemotherapeutic agent to induce a local effect and administra- tion of embolic materials to induce ischemic necrosis of the tumor cells; however, standardized techniques are lacking for each of the separate elements.

Conventional TACE (cTACE) consisting of administration of Lipiodol ${ }^{\circledR}$ (Guerbet LLC, Tokyo, Japan) and anthracycline agents, followed by embolization with gelatin sponge particles, has been a practical standard treatment in Asian countries [4]. Recently, the use of drug-eluting beads (DEB) with calibrated anthracycline drug-carrying microspheres (DC 


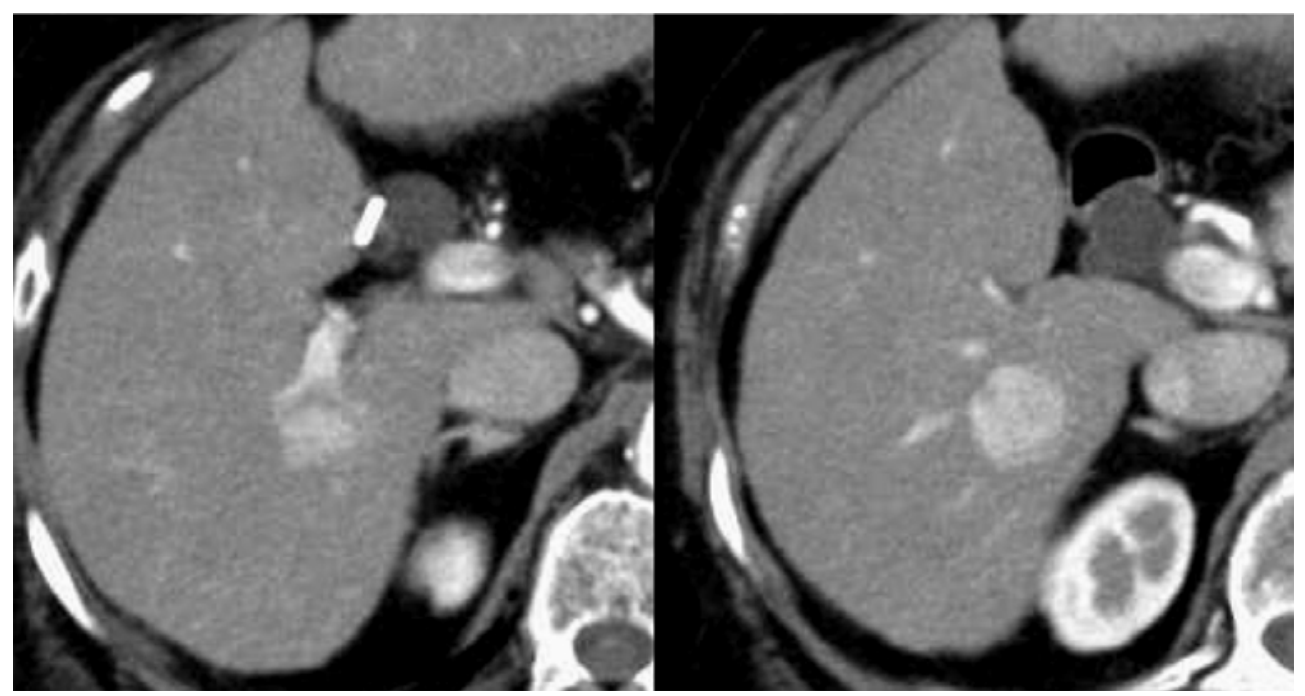

Figure 1. Imaging findings from a 79-year-old woman with hepatocellular carcinoma. In arterial phase of dynamic CT, a hypervascular tumor was observed in segment 6 of liver.

Bead; Eisai Co., Ltd., Tokyo, Japan) was introduced as a novel method capable of sustained and tumor-selective drug delivery and permanent embolization [5]. DEB has gained wide acceptance and has been increasingly used as the firstline TACE procedure in Western countries [6]. Four multicenter international randomized trials comparing DEB-TACE and cTACE demonstrated equivalent efficacy results [7-10]. Thus, controversy remains regarding the selection of the embolic materials and the techniques for TACE. In accordance with the approval of DEB in Japan in 2014, the Japan Interventional Radiology in Oncology Study Group (JIVROSG) aimed to conduct a prospective, multi-center RCT comparing DEB-TACE and cTACE employing a standard technique in Asian countries. Given that there were no available clinical data regarding DEB-TACE in patients of Japanese ethnicity, and the available drug is different from that used in previous studies because epirubicin is the only approved anthracycline drug for hepatic arterial infusion in Japan, it was necessary to evaluate the feasibility of DEB-TACE in Japanese patients prior to the initiation of the principal RCT. The purpose of the present study was to evaluate the feasibility of DEB-TACE with $75 \mathrm{mg}$ epirubicin for $\mathrm{HCC}$ in Japanese patients with unresectable HCC.

\section{Materials and Methods}

\section{Study Design}

This study was conducted as a prospective multi-center feasibility study.

\section{Patient Eligibility}

Eligible patients had unresectable Barcelona Clinic Liver Cancer (BCLC) stage A or B HCC that was unsuitable for curative treatments. Inclusion criteria were as follows: histologically or clinically diagnosed HCC excluding mixed type histology; unsuitability as a candidate for hepatic resection, liver transplantation, or local ablative therapy; hypervascular tumor showing enhancement in the early phase on computed tomography (CT) or magnetic resonance (MR) imaging (Fig. 1); no tumor thrombosis in the first branch or main portal vein; Eastern Cooperative Oncology Group performance status of 0-2; Child-Pugh classification of A or B; adequate hematologic, hepatic, renal, and cardiac function (leukocytes $\geq 3,000 / \mathrm{mm}^{3}$, platelets $\geq 50,000 / \mathrm{mm}^{3}$, serum bilirubin $\leq 3.0 \mathrm{mg} / \mathrm{dL}$ ); age $\geq 20$ years. Exclusion criteria were as follows: prior biliary-enteric bypass or endoscopic transampullary stent placement or percutaneous biliary drainage; refractory ascites or pleural effusion; severe arterioportal or arteriovenous shunts in the liver; allergy to contrast medium; severe and active comorbidity such as heart disease or renal disease; hepatic encephalopathy or severe mental disorder; active gastrointestinal bleeding; active concomitant malignancy; pregnancy, lactation, or childbearing potential.

The present study was compatible with the Health Insurance Portability and Accountability Act and approved by the institutional review boards of the participating institutions. Informed consent was obtained from all patients.

\section{DEB-TACE Procedure}

One vial of 100 to $300 \mu \mathrm{m}$ DEB was loaded with $75 \mathrm{mg}$ epirubicin. Epirubicin was chosen based on its use in cTACE in Asian countries and the approval status of anthracycline drugs for hepatic arterial infusion in Japan. The dose of epirubicin was determined based on recent studies [11-13] in which the anthracycline doxorubicin was used at a dose of $50 \mathrm{mg}$. We set the dose of epirubicin at $75 \mathrm{mg}$, which is equivalent in potency to $50 \mathrm{mg}$ of doxorubicin. Selective embolization using a microcatheter was performed according to the technical recommendations of Lencioni et al. [6]. DEB-TACE was performed as follows: (i) tumor en- 

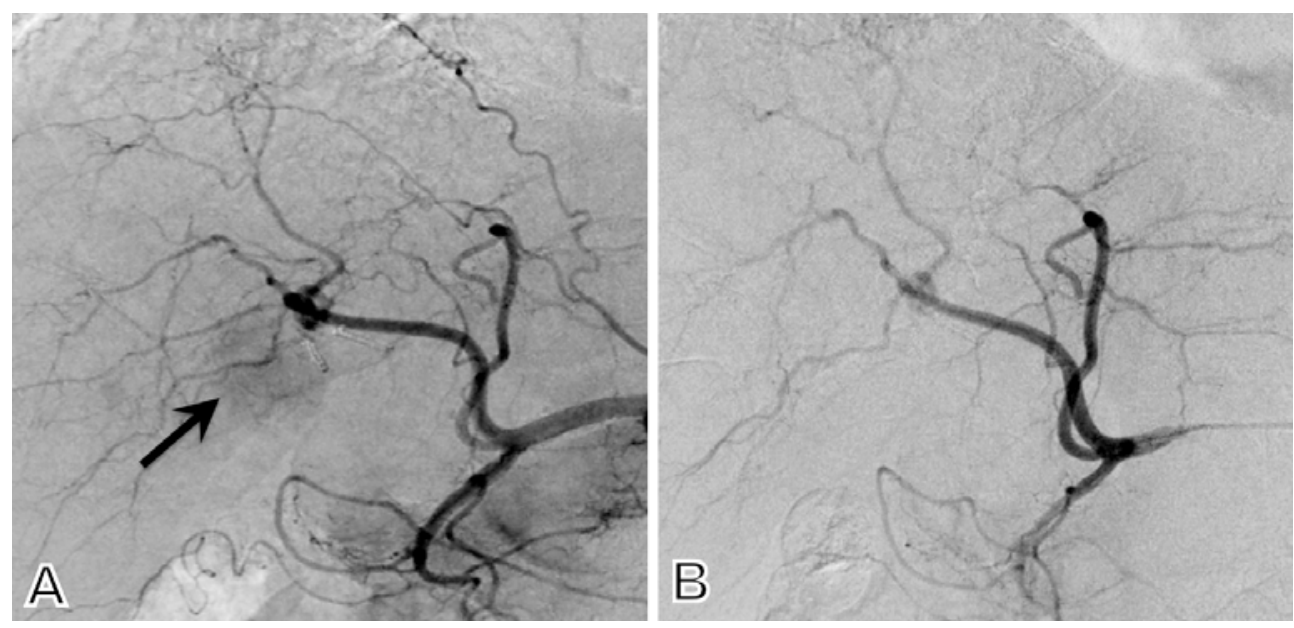

Figure 2. Angiography from the celiac trunk, a hypervascular tumor (arrow) was observed in right lobar of liver (A). The post-DEB-TACE angiography showed no tumor stain (B).

Table 1. Characteristics of Patients

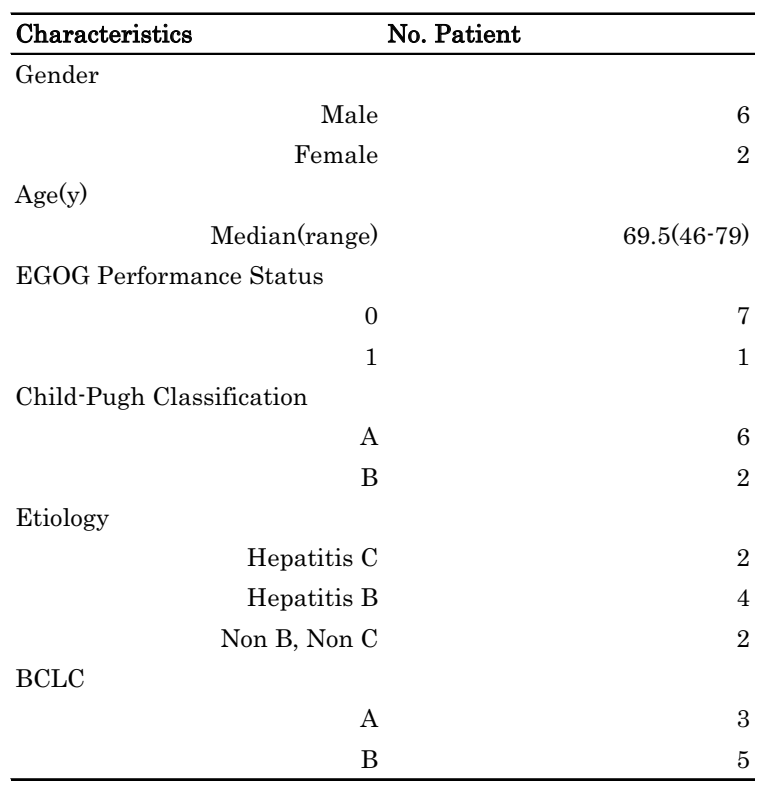

ECOG: Eastern Cooperative Oncology Group,

BCLC: Barcelona Clinic Liver Cancer

hancement and the feeding artery were confirmed using abdominal angiography; (ii) a microcatheter was inserted into the feeding artery of the HCC, followed by the injection of the DEB; and (iii) the injection was continued until near stasis was observed in the artery directly feeding the tumor (Fig. 2). Concurrent use of embolic agents other than DEB was not allowed.

\section{Study Design and Endpoints}

This study was designed as a prospective, nonrandomized study in two cancer center hospitals. The primary endpoint was the tumor response rate at 4 weeks after the procedure. Contrast-enhanced CT or MR imaging was performed 4 weeks after DEB-TACE. The tumor response was evaluated according to the modified Response Evaluation Criteria in Solid Tumors (m-RECIST) [14]. The secondary endpoint was the occurrence of any adverse event (AE). The severity of all AEs was evaluated according to the National Cancer Institute Common Terminology Criteria for Adverse Events (NCI CTCAE), version 4.0.

\section{Statistical Considerations}

We defined the feasibility of epirubicin DEB-TACE as the attainment of a clinical response in 2 or more patients out of the 8 who were enrolled. We set the threshold response rate of the main trial at $50 \%$. If a clinical response was observed in fewer than 2 of the 8 cases, the $95 \%$ confidence interval (CI) of the threshold response rate was below 50\%. In that case, the method would not be considered feasible and the principal RCT would have to be reconsidered.

\section{Results}

\section{Patient Characteristics}

Between May and August 2014, 8 patients from two institutions were enrolled in this clinical study. The characteristics of the patients are shown in Table 1. In brief, out of 8 enrolled patients, the Child-Pugh classification was $\mathrm{A}$ in 6 patients $(75.0 \%, 6 / 8)$, hepatitis etiology was hepatitis B virus in 3 patients $(37.5 \%, 3 / 8)$, and BCLC stage was $\mathrm{A}$ in 3 $(37.5 \%, 3 / 8)$ and $\mathrm{B}$ in 5 patients $(62.5 \%, 5 / 8)$.

\section{Tumor and Procedure Characteristics}

The characteristics of the tumors and procedures are listed in Table 2. Median maximum viable tumor size was 24.2 $\mathrm{mm}$ (standard deviation [SD]: $18.8 \mathrm{~mm}$ ). All patients had multiple tumors, and a bilobar distribution was observed in 6 patients $(75.0 \%, 6 / 8)$. All procedures were technically successful with a median number of vials of DEB of 0.43 (SD: 0.26). The mean dose of epirubicin was $38.1 \mathrm{mg}$ (SD: 19.7 $\mathrm{mg})$. 


\section{Tumor Response}

No instances of complete response were observed in any of the 8 patients, 4 patients showed a partial response (Fig. 3), 2 had stable disease, and 2 showed progressive disease. The overall response rate was 50\% (95\% CI: 22-79\%).

\section{Adverse Events}

The AEs associated with the DEB-TACE procedure in the present study are listed in Table 3. There were no grade 4 or higher AEs. Grade 3 thrombocytopenia occurred in 1 patient. One patient experienced a grade 3 increase in aspartate aminotransferase, alanine aminotransferase, and bilirubin levels. All AEs were managed with medical treatments. There were no procedure-related deaths.

\section{Discussion}

DEB-TACE with $75 \mathrm{mg}$ epirubicin was found to be feasible as treatment for unresectable HCC in the present study. Because the size of the cohort was small, the response rate

Table 2. Characteristics of Tumor and Procedure

\begin{tabular}{|c|c|c|}
\hline \multicolumn{3}{|c|}{ Maximum viable tumor size $(\mathrm{mm})$} \\
\hline & Median (range) & $24.2(19.5-80)$ \\
\hline \multicolumn{3}{|l|}{ No. of tumors } \\
\hline & Single & 0 \\
\hline & Multiple & 8 \\
\hline \multicolumn{3}{|l|}{ Tumor distribution } \\
\hline & Unilobar & 2 \\
\hline & Bilobar & 6 \\
\hline Technical success & & $8 / 8$ \\
\hline \multicolumn{3}{|l|}{ No. of DEB vial } \\
\hline & Median (range) & $0.43(0.25-1)$ \\
\hline
\end{tabular}

of $50 \%$ in this study cannot be precisely compared to the results from previous studies (Table 4); however, this rate is equivalent to the results of the PRECISION $\mathrm{V}$ study as reported by Lammer et al. Additionally, a $38.1 \mathrm{mg}$ dose of epirubicin, which has a titer 1.5 times higher than that of doxorubicin (corresponding value: approximately $57 \mathrm{mg}$ ), was equivalent to that in previous studies in which doses of doxorubicin ranging from 55.1 to $57.8 \mathrm{mg}$ were used. With regard to safety, no severe AEs were observed in the present study, consistent with the results of previous studies. Thus, it is deemed that a dose of $75 \mathrm{mg}$ epirubicin per vial of DC Beads is acceptable in terms of safety and efficacy. The performance of DEB-TACE has increased in many institutions across the country following the approval of DC Beads in Japan in 2014. However, the dose of epirubicin has not been standardized, and doses vary across institutions. Therefore, the results presented here (75 $\mathrm{mg}$ epirubicin per vial) may provide a standard for the loading dose.

In the previous RCTs comparing cTACE with DEB-TACE in Western countries, cTACE techniques utilized various doses of chemotherapeutic agents (doxorubicin 56.9-223 $\mathrm{mg}$ ), various embolic materials, and scheduled treatment timing. There has been no standardization of the technical procedure for cTACE. Accordingly, the authors determined that we needed to conduct a prospective, multi-center RCT comparing DEB-TACE and cTACE employing a standard technique in Asian countries. Asian cTACE consists of selective administration of the chemotherapeutic agents (approximately $40-60 \mathrm{mg}$ of doxorubicin or epirubicin) mixed with Lipiodol into the feeding branch of the tumor followed by embolization with a gelatin sponge. The timing of the next treatment is unpredictable, depending on demands determined by the clinical and imaging findings of residual or recurrent tumors. The rationale of the safety and efficacy data of DEB-TACE has not been established in Japan. Data

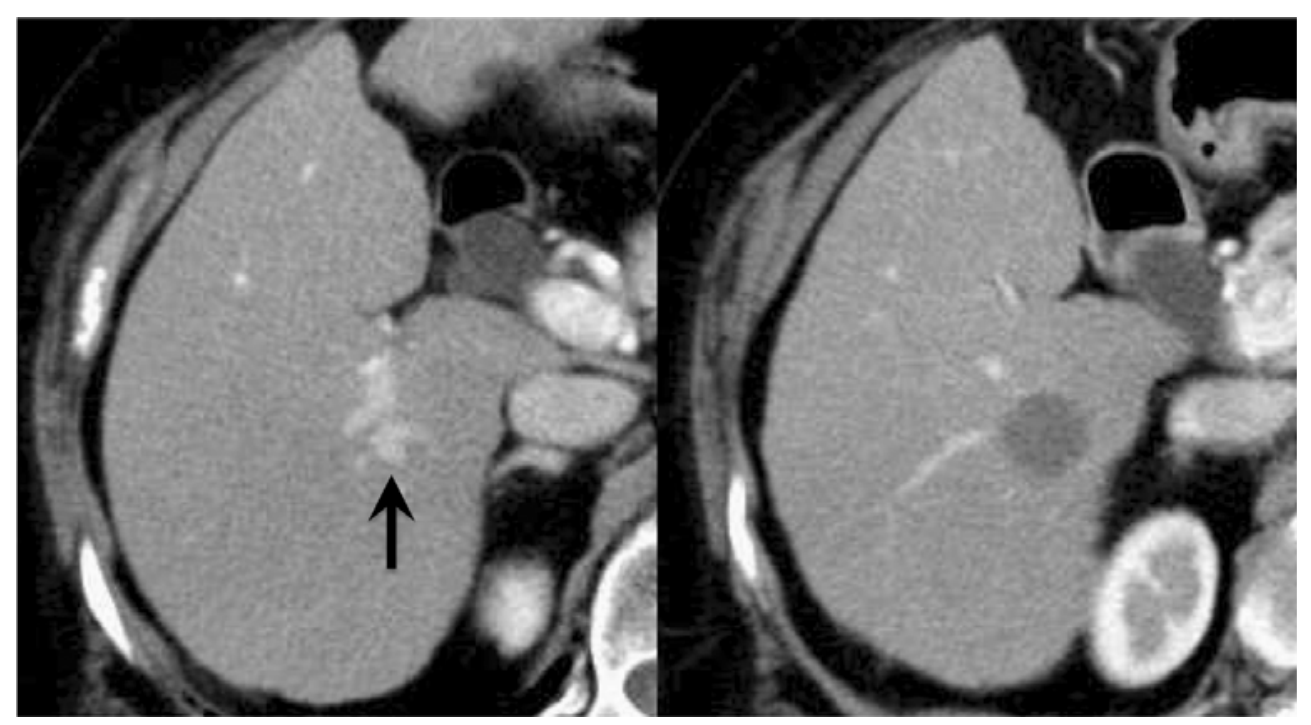

Figure 3. CT at four weeks after DEB-TACE demonstrated disappearance of early enhancement in most of the tumor. Small enhanced area corresponding to viable hepatocellular carcinoma was seen in the cranial part of the tumor (arrow). This case was regarded as partial response. 
Table 3. Adverse Events

\begin{tabular}{cccc}
\hline AEs & Grade 1 & Grade 2 & Grade 3 \\
\hline Fatigue & 1 & 2 & 0 \\
Fever & 2 & 0 & 0 \\
Anorexia & 1 & 1 & 0 \\
Nausea & 1 & 0 & 0 \\
Vomiting & 3 & 0 & 0 \\
Alopecia & 0 & 0 & 0 \\
Platelets & 0 & 2 & 1 \\
WBC & 0 & 1 & 0 \\
AST & 0 & 1 & 1 \\
ALT & 0 & 1 & 1 \\
Bilirubin & 0 & 1 & 1 \\
\hline
\end{tabular}

WBC: white blood cell, ALT: alanine aminotransferase,

AST: aspartate aminotransferase.

Grading according to Common Terminology Criteria for Adverse Events, version 4.0

Table 4. Randomized Controlled Trials of Transcatheter Arterial Embolization

\begin{tabular}{|c|c|c|c|c|c|c|c|}
\hline Author, Year & Study Design & Treatment & Drug & $\begin{array}{l}\text { Mean dose of drug } \\
(\mathrm{mg})\end{array}$ & No. Patient & $\begin{array}{l}\text { Response } \\
\text { Rate }\end{array}$ & Summary of AEs \\
\hline \multirow{2}{*}{$\begin{array}{l}\text { Lammer, } 2010 \\
\text { (PRECISION V) }\end{array}$} & \multirow[t]{2}{*}{ Phase 2, RCT } & conventional TACE & doxorubicin & 223 & 108 & $44 \%(6 \mathrm{M})$ & $\begin{array}{c}\text { Alopecia } \\
\text { Marrow suppression } \\
\text { Mucositis }\end{array}$ \\
\hline & & DEB-TACE & doxorubicin & 295 & 93 & $52 \%(6 \mathrm{M})$ & Marrow suppression \\
\hline \multirow{3}{*}{ Sacco, 2011} & \multirow{3}{*}{ Phase 2, RCT } & conventional TACE & doxorubicin & 56.9 & 34 & $100 \%(1 \mathrm{M})$ & Cholecystitis \\
\hline & & & & & & & \\
\hline & & DEB-TACE & doxorubicin & 55.1 & 33 & $100 \%(1 \mathrm{M})$ & Liver failure \\
\hline \multirow{2}{*}{$\begin{array}{c}\text { Van Malenstein, } \\
2011\end{array}$} & \multirow{2}{*}{ Phase 2, RCT } & conventional TACE & doxorubicin & $68\left(/ \mathrm{m}^{2}\right)$ & 14 & $92 \%(6 \mathrm{~W})$ & $\begin{array}{c}\text { Alopecia } \\
\text { Leukopenia } \\
\text { Liver dysfunction }\end{array}$ \\
\hline & & DEB-TACE & doxorubicin & $65\left(/ \mathrm{m}^{2}\right)$ & 16 & $77 \%(6 \mathrm{~W})$ & $\begin{array}{c}\text { Infection } \\
\text { Liver dysfunction } \\
\text { Cholecystitis }\end{array}$ \\
\hline \multirow{2}{*}{$\begin{array}{c}\text { Golfieri, } 2014 \\
\text { (PRECISION Italia) }\end{array}$} & \multirow{2}{*}{ Phase 2, RCT } & conventional TACE & epirubicin & 47.2 & 86 & $89 \%(1 \mathrm{M})$ & $\begin{array}{c}\text { Post-procedual pain } \\
\text { Infection } \\
\text { Liver function worsening }\end{array}$ \\
\hline & & DEB-TACE & doxorubicin & 57.8 & 88 & $90 \%(1 \mathrm{M})$ & $\begin{array}{c}\text { Fever } \\
\text { Pain } \\
\text { Cholecystitis } \\
\end{array}$ \\
\hline
\end{tabular}

RCT: randomized control trial, TACE: transarterial chemoembolization, DEB: drug-eluting beads, AEs: adverse events

from the present study are preliminary; however, it is acceptable to affirm the feasibility of $75 \mathrm{mg}$ epirubicin DEBTACE in the Japanese population. Thus, based on the data from the present study, our study group has planned to conduct a prospective RCT comparing DEB-TACE and cTACE.

This study has several limitations. First, evaluation of the response was only conducted at one point (4 weeks after the treatment). This duration was determined based on the first evaluation point at approximately 1 month after TACE used in previous studies. Second, there may have been a lack of experience with the use of DEB because this study was conducted shortly after the approval of DEB in Japan. However, the use of a microcatheter in the present study was the same technique employed in cTACE in our daily clinical practice. Lectures as well as practical training regarding the best practices of embolization, including optimized preparation and embolization endpoints, were provided during a workshop prior to our first use of DEB.

In conclusion, DEB-TACE with $75 \mathrm{mg}$ epirubicin was feasible in Japanese patients and it was deemed appropriate to proceed to an RCT comparing DEB-TACE and cTACE.

This study has been presented at WCIO 2015.

Conflict of interest: There are no potential conflicts of interest with regard to this study.

Acknowledgement: This study was supported in part by Grantsin-Aid for Cancer Research from the Ministry of Health, Labour, and Welfare of Japan and the National Cancer Center Research and Development Fund.

\section{References}

1. Lo CM, Ngan H, Tso WK, Liu CL, Lam CM, Poon RT, et al. Randomized controlled trial of transarterial lipiodol chemoembolization for unresectable hepatocellular carcinoma. Hepatology 2002; 35:1164-1171.

2. Llovet JM, Real MI, Montana X, Planas R, Coll S, Aponte J, et al. Arterial embolisation or chemoembolisation versus symptomatic treatment in patients with unresectable hepatocellular carcinoma: a randomised controlled trial. Lancet 2002; 359:1734-1739. 
3. Llovet JM, Bruix J. Systematic review of randomized trials for unresectable hepatocellular carcinoma: Chemoembolization improves survival. Hepatology 2003; 37:429-442.

4. Ikeda M, Arai Y, Park SJ, Takeuchi Y, Anai H, Kim JK, et al. Prospective study of transcatheter arterial chemoembolization for unresectable hepatocellular carcinoma: an Asian cooperative study between Japan and Korea. J Vasc Interv Radiol 2013; 24:490-500.

5. Varela M, Real MI, Burrel M, Forner A, Sala M, Brunet M, et al. Chemoembolization of hepatocellular carcinoma with drug eluting beads: efficacy and doxorubicin pharmacokinetics. J Hepatol 2007; 46:474-481.

6. Lencioni R, de Baere T, Burrel M, Caridi JG, Lammer J, Malagari $\mathrm{K}$, et al. Transcatheter treatment of hepatocellular carcinoma with Doxorubicin-loaded DC Bead (DEBDOX): technical recommendations. Cardiovasc Intervent Radiol 2012; 35:980-985.

7. Lammer J, Malagari K, Vogl T, Pilleul F, Denys A, Watkinson A, et al. Prospective randomized study of doxorubicin-eluting-bead embolization in the treatment of hepatocellular carcinoma: results of the PRECISION V study. Cardiovasc Intervent Radiol 2010; 33:41-52.

8. Golfieri R, Giampalma E, Renzulli M, Cioni R, Bargellini I, Bartolozzi C, et al. Randomised controlled trial of doxorubicin-eluting beads vs conventional chemoembolisation for hepatocellular carcinoma. Br J Cancer 2014; 111:255-264.
9. Sacco R, Bargellini I, Bertini M, Bozzi E, Romano A, Petruzzi P, et al. Conventional versus doxorubicin-eluting bead transarterial chemoembolization for hepatocellular carcinoma. J Vasc Interv Radiol 2011; 22:1545-1552.

10. van Malenstein H, Maleux G, Vandecaveye V, Heye S, Laleman $\mathrm{W}$, van Pelt $\mathrm{J}$, et al. A randomized phase II study of drug-eluting beads versus transarterial chemoembolization for unresectable hepatocellular carcinoma. Onkologie 2011; 34:368-736.

11. Padia SA, Shivaram G, Bastawrous $S$, Bhargava P, Vo NJ, Vaidya $\mathrm{S}$, et al. Safety and efficacy of drug-eluting bead chemoembolization for hepatocellular carcinoma: comparison of small-versus medium-size particles. J Vasc Interv Radiol 2013; 24:301-306.

12. Prajapati HJ, Dhanasekaran R, El-Rayes BF, Kauh JS, Maithel SK, Chen Z, et al. Safety and efficacy of doxorubicin drug-eluting bead transarterial chemoembolization in patients with advanced hepatocellular carcinoma. J Vasc Interv Radiol 2013; 24:307-315.

13. Kogut MJ, Chewning RH, Harris WP, Hippe DS, Padia SA. Postembolization syndrome after hepatic transarterial chemoembolization: effect of prophylactic steroids on postprocedure medication requirements. J Vasc Interv Radiol 2013; 24:326-331.

14. Lencioni R, Llovet JM. Modified RECIST (mRECIST) assessment for hepatocellular carcinoma. Semin Liver Dis 2010; 30:52-60. 